\title{
A REVIEW PAPER: DESIGN AND DEVELOPMENT OF AUTOMATIC INSPECTION SYSTEM FOR DIMENSIONAL MEASUREMENT OF MACHINED AND FORGED COMPONENTS FOR INDUSTRIAL APPLICATION USING IMAGE PROCESSING
}

\author{
Ms. Anuradha Manik Kambale \\ Department of Electronics Engineering \\ AITRC, Vita, Maharashtra, India
}

\author{
Dr.S.S Patil \\ Department of Electronics Engineering \\ KBPCOE, Satara Maharashtra, India
}

\begin{abstract}
With increased demand for forged and machined product of high quality and safety standards, that needs accurate, fast and objective quality determination of these products. Computer vision provides an automated and cost-effective technique to accomplish the requirements of customers. This inspection approach based on image processing has found a variety of different applications in the various types of forged industry. Considerable research has highlighted its potential and importance. Machine vision has been successfully adopted for the quality analysis and inspection. From the study of literature papers it has observed that it has observed that most of the forging industry used image processing technology for better results.
\end{abstract}

Keywords - quality, forged components, machined components

\section{INTRODUCTION}

In current global manufacturing industries, quality of the merchandise is becoming necessity and demands high excatitude with accuracy for every item. Quality review is nothing but an activity which consist measuring, examining or testing the product characteristics and comparing the result with specific requirement given by the customer or preset standards to ensure the conformity achieved for each characteristic. Manufacturing product exhibits several quality characteristics. Quality inspection becomes a big way to verify product uniformity to requirements. Under certain review procedures, here we may identify whether the product quality matches to given specifications or not. If the product quality fails to obey many possible reasons come include problems from the earlier manufacturing process. Current industries need efficient quality inspection systems to ensure their products are free of defects and they are as per given standards or requirements.[3-4].

Evolution in industry, increasing complexity of quality requirements, customers demand a higher level of product quality. Manufacturing industry look at some of the challenges as they have to deliver product at consistently high-quality standards, navigate end-to-end supply chains and manage strict time-to-market deadlines driven by demanding customers. Since manufacturing industries are highly sensitive to time, cost, and quality. Most of the industry uses automation which may help fill the labor gap, skilled workers will hamper the performance analysis and production.[3] Comparing the performance of large automated industry and small-scale industry most of the small industry uses a traditional manual measurement method cannot guarantee product quality. Therefore, it is of great significance to improve the technology level of the small-scale manufacturing industry with low cost, high precision, and high efficiency automatic system

To inspect a product characteristic by traditional measurement technique, need wide range of measuring equipment's and this process is time consuming. Manual measurement method done by the inspector have chances of human error and it not precise activity. [1-2] It becomes very much difficult when it comes with wide range of products with large quantity in mass or batch production. This might affect the delivery and result which confirms the quality requirement which are previously set.

\section{LITERATURE REVIEW}

The literature has come out with several approaches of quality inspection system. The review has been collected as follows.

1.Nilesh P. Vanikar Dr. Shantipal S. Ohol Rajendra Anjikar, [2015][1] defects related to manual operations in first coat 
shelling and pouring are discussed. The defects are mainly originated due to inconsistency of the process due to human mistakes; safety major's etc. also provide the need of automation in investment casting industry due to variety of defects caused by manual operations.

2.Mithun Gaur, [2019][2] Provides the need of digital transformation importance of and modernization in industry with future aspect. Preparing the future change and building innovative solutions leveraging digital technologies.

3.Marek Hawryluk, Jacek Ziemba, Przemysław Sadowski, [2017][3] presents the possibilities of applying modern measuring techniques, classic and coordinate measuring techniques in the forging industry (various stages of technological sequence), with the use of various measuring equipments, instruments and machines applied in workshop calibration.

4.Li Bin, September 2018 [4] uses the optical instrument geometric measurement technology is unwieldy in reading process, the introduction of machine vision technology into geometric dimensional measurement can achieve quick measurement of the dimension or relative position of manufactured objects (products or parts). It can achieve realtime online, non-contact, fast speed, good flexibility, and high precision and high accuracy. It saves time and power and can avoid human mistakes in the process of dimensional measurement and can also achieve continuity of production rate and improve the automation level of manufacturing of products.

5.Andrzej Sioma,2020[5] method of constructing a 3D image of the tile surface using the Laser Triangulation Method (LTM), along with the surface imaging parameters employed in the research. The algorithms of surface image analysis of ceramic tiles used in the process of image enhancement and defect identification are available on surface of products. For selected defects, the method of measuring defective parameters and the method of visualization of various types of the surface defects are also presented.

6.Qingsheng Jiang, Dapeng Tan , Yanbiao Li, Shiming Ji , Chaopeng Cai Qiming Zhen,2020 [6] deep learning method based on convolutional neural network feature extraction is used to find band analyse the components detection and classification of various metal shaft surface defects.

7.Jia, H.B.; Murphey, Y.L.; Shi, J.J,2004 [7] developed a machine learning system based on the Support Vector Machine. During the design of the machine vision system, we address three main requirements: (1) it should be robust and capable of providing good discrimination even in the case of noisy input data. (2) It must be fast enough in order to meet inline speed requirements. (3)

\section{OBJECTIVES OF STUDY}

In the current scenario, quality improvement is a highly desired objective in the competitive international business world. This study relates alternative quality approaches to define better the relationship between quality and productivity. The objectives of proposed research are listed below

1. To study problems faced by manufacturing industry.

2. To Review and analyze the traditional technology of automatic quality inspection system.

3. To Review and analyze the various methods of image acquisition system as well as sensors.

4. To Understand different aspect of quality inspection.

5. To Study and evaluation of various surface as well as geometric defects of forged and machined components

6. To Creating cost effective model for precise measurement and better accuracy.

7. To provide faster feedback to upstream production units to reduce the rejection of products.

\section{PROBLEM STATEMENT}

It is a common practice in industrial manufacturing sector the use manually operated instruments for dimensional inspection which is costlier and time consuming which affect the overall industrial manufacturing process. In this regard it is a proposal for development of automatic dimensional measurement system to ensure the conformity of desired specifications which will be quick and more precise for better product quality.

\section{METHODOLOGY}

For the industrial quality automation, it is very important to identify the desired and the opportunity of the system to be fully automated. The production cost, and involvement of the machines, the utility requirement of the machines, quality parameters of the products are most important factors to consider while planning for quality automation. The system that has flexibility, ease of programming, adaptability to change, expandability, enhance ability of function, ruggedness in system, service back up. Performance factor for automation system are response time, reliability, maintainability, availability and capability etc. Automation is one of the most effective system for reduction in cost, waste, scrap, labor, time, and controlling quality, and improving overall performance of any machine, system or process

\section{CONCLUSION}

This paper covers the fundamental concept and importance of quality inspection based on image processing. In today's world each and every automated industry uses image processing technology for quality inspection. Also this paper focuses on 
the importance of quality inspection used for forged and machined components based on image processing technology. In future as evolution in industrial automization sector goes on the more will be use of advanced technology for reliable, effective and accurate inspection system.

\section{REFERENCES}

[1] Nilesh P. Vanikar Dr. Shantipal S. Ohol Rajendra Anjikar," Need of Automation in Investment Casting Industry Due to Major Defects Caused by Manual Operations", International Journal for Scientific Research \& Development| Vol. 3, Issue 09, 2015

[2] Mithun Gaur," Digital transformation framework for driving future growth", International Journal of Advanced Research in Computer Science \& Technology,2019

[3] Marek Hawryluk, Jacek Ziemba, Przemysław Sadowski," A Review of Current and NewMeasurement Techniques Used in Hot Die Forging Processes", Measurement and Control 2017,

Vol. 50(3) 74-86

[4] Li Bin, "Research on geometric dimension measurement system of shaft parts based on machinevision", EURASIP Journal on Image and Video Processing, September (2018)

[5] Andrzej Sioma,"Automated Control of Surface Defects on Ceramic Tiles Using 3D Image Analysis", Materials , March 2020

[6] Qingsheng Jiang, Dapeng Tan , Yanbiao Li, Shiming Ji , Chaopeng Cai and Qiming Zheng," Object Detection and Classification of Metal Polishing Shaft Surface Defects Based on Convolutional Neural Network Deep Learning", Appl. Sci. 2020,

[7] Jia, H.B.; Murphey, Y.L.; Shi, J.J. An intelligent real-time vision system for surface defect detection.In Proceedings of the 17th International Conference on Pattern Recognition, Cambridge, UK, 23-26 August 2004; pp. 239-242.

[8] Huda M. Jawad \& Tahseen A. Husain,' Measuring Object Dimensions and its Distances BasedImage Processing Technique by Analysis the Image Using Sony Camera", Eurasian Journal of Science \& Engineering ISSN 2414-5629 (Print), ISSN 2414-5602 (Online), October 2017
[9] S Howimanporn, S Chookaew" Position Measurement System Based on Image Trajectory Tracking Control of Directional Conveyor", International Conference on Computer and Electrical Engineering Journal of Physics: Conference Series ,2017 